\title{
Mutually exclusive mutations in NOTCH1 and PIK3CA associated with clinical prognosis and chemotherapy responses of esophageal squamous cell carcinoma in China
}

Bin Song ${ }^{1,2,3, *}$, Heyang Cui ${ }^{1,2, *}$, Yaoping Li ${ }^{1,4, *}$, Caixia Cheng ${ }^{1,2,5, *}$, Bin Yang ${ }^{1,4, *}$, Fang Wang $^{1,2, *}$, Pengzhou Kong ${ }^{1,2, *}$, Hongyi Li ${ }^{1,2}$, Ling Zhang ${ }^{1,2}$, Zhiwu Jia ${ }^{1,2}$, Yanghui Bi ${ }^{1,2}$, Jiaqian Wang ${ }^{6}$, Yong Zhou ${ }^{6}$, Jing Liuu, ${ }^{1,7}$, Juan Wang ${ }^{1,2}$, Zhenxiang Zhao ${ }^{1,2}$, Yanyan Zhang ${ }^{1,7}$, Xiaoling Hu${ }^{1,2}$, Ruyi Shi ${ }^{1,2}$, Jie Yang ${ }^{1,2}$, Haiyan Liu ${ }^{1,2,8}$, Ting Yan ${ }^{1,2}$, Yike $\mathbf{L i}^{1,7}$, Enwei Xu ${ }^{1,2,9}$, Yu Qian ${ }^{1,2}$, Yanfeng $\mathbf{X i}^{9}$, Shiping Guo ${ }^{4}$, Yunqing Chen ${ }^{4}$, Jinfen Wang 9 , Guodong Li ${ }^{9}$, Jianfang Liang ${ }^{5}$, Junmei Jia ${ }^{3}$, Xing Chen ${ }^{10}$, Jiansheng Guo ${ }^{7}$, Tong Wang ${ }^{11}$, Yanbo Zhang ${ }^{11}$, Qingshan Li ${ }^{12}$, Chuangui Wang ${ }^{13}$, Xiaolong Cheng ${ }^{1,2}$, Qimin Zhan ${ }^{14}$ and Yongping Cui ${ }^{1,2}$

${ }^{1}$ Translational Medicine Research Center, Shanxi Medical University, Taiyuan, Shanxi, China

${ }^{2}$ Key Laboratory of Cellular Physiology, Ministry of Education, Shanxi Medical University, Taiyuan, Shanxi, China

${ }^{3}$ Department of Oncology, The First Hospital, Shanxi Medical University, Taiyuan, Shanxi, China

${ }^{4}$ Department of Tumor Surgery, Shanxi Cancer Hospital, Taiyuan, Shanxi, China

${ }^{5}$ Department of Pathology, The First Hospital, Shanxi Medical University, Taiyuan, Shanxi, China

${ }^{6}$ BGI-Shenzhen, Shenzhen, Guangdong, China

7 Department of General Surgery, the First Hospital, Shanxi Medical University, Taiyuan, Shanxi, China

${ }^{8}$ Department of Nuclear medicine, the First Hospital, Shanxi Medical University, Taiyuan, Shanxi, China

${ }^{9}$ Department of Pathology, Shanxi Cancer Hospital, Taiyuan, Shanxi, China

${ }^{10}$ Department of Endoscopy, Shanxi Provincial People's Hospital, Taiyuan, Shanxi, China

11 Department of Statistics, Shanxi Medical University, Taiyuan, Shanxi, China

${ }^{12}$ School of Pharmaceutical Sciences, Shanxi Medical University, Taiyuan, Shanxi, China

${ }^{13}$ Key Laboratory of Medical Cell Biology, College of Translational Medicine, China Medical University, Shenyang, China

14 State Key Laboratory of Molecular Oncology, Cancer Institute and Cancer Hospital, Chinese Academy of Medical Sciences and Peking Union Medical College, Beijing, China

* Authors share co-first authorship

Correspondence to: Yongping Cui, email: cuiy0922@yahoo.com

Qimin Zhan, email: zhanqimin@pumc.edu.cn

Keywords: esophageal cancer, significantly mutated genes, mutational exclusivity, oncogene

Received: August 05, $2015 \quad$ Accepted: September 24, $2015 \quad$ Published: October 29, 2015

This is an open-access article distributed under the terms of the Creative Commons Attribution License, which permits unrestricted use, distribution, and reproduction in any medium, provided the original author and source are credited.

\section{ABSTRACT}

Background: Recurrent genetic abnormalities that correlate with clinical features could be used to determine patients' prognosis, select treatments and predict responses to therapy. Esophageal squamous cell carcinoma (ESCC) contains genomic alterations of undefined clinical significance. We aimed to identify mutually exclusive mutations that are frequently detected in ESCCs and characterized their associations with clinical variables.

Methods: We analyzed next-generation-sequencing data from 104 ESCCs from Taihang Mountain region of China; 96 pairs were selected for deep target-capturebased validation and analysis of clinical and pathology data. We used model proposed by Szczurek to identify exclusive mutations and to associate these with pathology findings. Univariate and multivariate analyses with Cox proportional hazards model 
were used to examine the association between mutations and overall survival and response to chemotherapy. Findings were validated in an analysis of samples from 89 patients with ESCC from Taihang Mountain.

Results: We identified statistically significant mutual exclusivity between mutations in NOTCH1 and PIK3CA in ESCC samples. Mutations in NOTCH1 were associated with well-differentiated, early-stage malignancy and less metastasis to regional lymph nodes. Nonetheless, patients with NOTCH1 mutations had shorter survival times than patients without NOTCH1 mutations, and failed to respond to chemotherapy. In contrast, patients with mutations in PIK3CA had better responses to chemotherapy and longer survival times than patients without PIK3CA mutations. Conclusions: In a genetic analysis of ESCCs from patients in China, we identified mutually exclusive mutations in NOTCH1 and PIK3CA. These findings might increase our understanding of ESCC development and be used as prognostic factors.

\section{INTRODUCTION}

Esophageal cancer causes 400,200 deaths each year and represents the sixth leading cause of cancer deaths worldwide [1]. It is categorized into esophageal adenocarcinoma and esophageal squamous cell carcinoma (ESCC) [1]. China has the highest incidence and mortality rate of ESCC in the world; approximately $70 \%$ of worldwide ESCC cases occur in China, where it is ranked the fourth most lethal cancer [2]. The risk of developing ESCC in China has been linked to factors such as gender, dietary habits, and family history. Alcohol abuse and tobacco consumption represent minor factors in highrisk populations in China [2]. Currently, the treatment of ESCC relies on surgery, chemotherapy, radiotherapy, or combinations of these [3]. Although many reports have indicated that patients who receive chemoradiation therapy had a significantly better 5-year survival rate than patients who did not, it is unclear why certain patients respond better than others to chemotherapy [3]. It is common for patients to be either under- or over-treated in China, which results in increased chemoresistance or treatment toxicity [4]. Therefore, unambiguous molecular markers are needed to identify which patients are likely to respond best to particular treatments, and thereby avoid undertreatment or over-treatment .

ESCC has a striking geographic distribution worldwide, with a higher prevalence in certain restricted areas of China [5]. Specifically, Shanxi, Henan, and Hebei provinces (the Taihang Mountains in North-Central China) are the so-called "esophageal cancer belt" with extremely high incidences of ESCC compared with the elsewhere in China and the rest of the world (Supplementary Figure 1); over $20 \%$ of all deaths in this area have been attributed to this cancer [5].

Epidemiological and etiological studies have shown that environmental and genetic factors play crucial roles in esophageal carcinogenesis [6]. Recently, genome-wide investigations have characterized genomic alterations in ESCC [7-9]. For these next-generation sequencing (NGS) studies, patients were enrolled from either Chaoshan
District, Southern China [7] or the Cancer Institute and Hospital, Chinese Academy of Medical Sciences (CICAMS), where patients come from all over the country $[8,9]$. Moreover, we previously performed an NGS study on 104 ESCC patients who were recruited from Shanxi and Henan provinces, combined with mutation set of 17 whole-genome sequencing (WGS) and 71 whole-exome sequencing (WES) from Chaoshan District and reported their mutational signatures and identified driver genes or pathways that contributed to ESCC [10]. In large collections of tumor samples, it has been observed that alterations that affect the same cancer pathways tend to not co-occur in the same patient. Mutual exclusivity may arise, because alteration of only one pathway component is sufficient to deregulate the entire process. Such mutually exclusive alterations have frequently been observed and have been associated with pathological and clinical features; thus, they can serve as molecular markers for potential categorization of tumors and prediction of the prognosis and treatment response of patients [11, 12]. Detecting such patterns is important for understanding cancer progression and prognosis and could suggest genes for targeted treatment or markers for the improvement of treatment in patients [13]. Some mutually exclusive alterations in the genomes of ESCC patients have been identified but not studied very much, and no reliable molecular markers for diagnosing or predicting patient outcomes have been identified for this disease [8,9].

In this study, we describe the identification and validation of mutually exclusive mutational patterns of NOTCHI and PIK3CA, two significantly mutated genes (SMGs) identified in ESCC via genomic analyses [7-10], and their associations with clinical variables.

\section{RESULTS}

\section{Mutual exclusivity among SMGs in ESCC}

SMGs that possess a higher mutation rate than the expected background mutation rate may be positive 
Table 1: Summary of clinical characteristics of ESCC patients with NOTCH1 and PIK3CA mutations in cohort \#1.

\begin{tabular}{|c|c|c|c|c|c|c|c|c|c|}
\hline \multirow{2}{*}{\multicolumn{2}{|c|}{$\begin{array}{l}\text { Clinical, epidemiological } \\
\text { or pathological feature }\end{array}$}} & \multicolumn{4}{|c|}{ NOTCH1 } & \multicolumn{4}{|l|}{ PIK3CA } \\
\hline & & Total(N) & Mutant & Wild-type & $P$ value & Total(N) & Mutant & Wild-type & $P$ value \\
\hline \multicolumn{2}{|l|}{ All cases } & 104 & 22 & 82 & & 104 & 17 & 88 & \\
\hline \multirow{3}{*}{ Age } & $<60$ & 50 & 8 & 42 & \multirow{3}{*}{0.302} & 50 & 5 & 45 & \multirow{3}{*}{0.199} \\
\hline & $60-69$ & 40 & 12 & 28 & & 40 & 7 & 33 & \\
\hline & $\geq 70$ & 14 & 2 & 12 & & 14 & 4 & 10 & \\
\hline \multirow{2}{*}{ Sex } & Male & 104 & & & & 104 & & & \\
\hline & Female & 0 & & & & 0 & & & \\
\hline \multirow{2}{*}{$\begin{array}{l}\text { Tobacco } \\
\text { use }\end{array}$} & Yes & 96 & 19 & 77 & \multirow{2}{*}{0.467} & 96 & 15 & 81 & \multirow{2}{*}{1} \\
\hline & No & 8 & 3 & 5 & & 8 & 1 & 7 & \\
\hline \multirow{2}{*}{ Alcohol consumption } & Yes & 63 & 11 & 52 & \multirow{2}{*}{0.253} & 62 & 9 & 53 & \multirow{2}{*}{0.766} \\
\hline & No & 41 & 11 & 30 & & 42 & 7 & 35 & \\
\hline \multirow{2}{*}{ Family cancer history } & Yes & 17 & 5 & 12 & \multirow{2}{*}{0.557} & 17 & 2 & 15 & \multirow{2}{*}{1} \\
\hline & No & 87 & 17 & 70 & & 87 & 14 & 73 & \\
\hline \multirow{3}{*}{ Tumor location } & Upper thoracic & 4 & 0 & 4 & \multirow{3}{*}{0.027} & 4 & 3 & 1 & \multirow{3}{*}{0.006} \\
\hline & Middle thoracic & 42 & 4 & 38 & & 42 & 3 & 39 & \\
\hline & Lower thoracic & 57 & 17 & 40 & & 57 & 10 & 47 & \\
\hline \multirow{3}{*}{ Histological grade } & Grade 1 & 59 & 20 & 39 & \multirow{3}{*}{0.001} & 59 & 10 & 49 & \multirow{3}{*}{0.328} \\
\hline & Grade 2 & 33 & 0 & 33 & & 33 & 6 & 27 & \\
\hline & Grade 3 & 12 & 2 & 10 & & 12 & 0 & 12 & \\
\hline \multirow{3}{*}{ Pathologic Stage } & $\mathrm{I}$ & 60 & 18 & 42 & \multirow{3}{*}{0.011} & 51 & 8 & 43 & \\
\hline & II & & & & & & & & 0.933 \\
\hline & III & 44 & 4 & 40 & & 53 & 8 & 45 & \\
\hline & $\mathrm{T} 1$ & 4 & 3 & 1 & & 4 & 0 & 4 & \\
\hline Pathologic & $\mathrm{T} 2$ & 23 & 6 & 17 & & 23 & 5 & 18 & \\
\hline T stage & T3 & 72 & 13 & 59 & 0.004 & 72 & 10 & 62 & 0.604 \\
\hline & $\mathrm{T} 4$ & 5 & 0 & 5 & & 5 & 1 & 4 & \\
\hline Pathologic & 0 & 54 & 18 & 36 & DOPO & 54 & 9 & 45 & \\
\hline N stage & 1 & 50 & 4 & 46 & 0.002 & 50 & 7 & 43 & 0.100 \\
\hline & Yes & 39 & 12 & 27 & & 39 & 3 & 36 & \\
\hline & No & 56 & 10 & 46 & & 65 & 13 & 52 & \\
\hline & Failure & 39 & 11 & 28 & & 39 & 3 & 36 & \\
\hline & effective & 31 & 5 & 26 & & 31 & 10 & 21 & \\
\hline 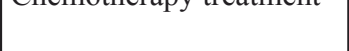 & No treatment & 24 & 4 & 20 & & 24 & 3 & 21 & $0.02 \mathrm{~J}$ \\
\hline & missing & 10 & 1 & 9 & & 10 & 0 & 10 & \\
\hline & Dead & 45 & 8 & 37 & & 45 & 4 & 41 & \\
\hline Proonosis & Survival & 41 & 11 & 30 & & 41 & 12 & 29 & \\
\hline Mantel-Cox test) & Missing & 18 & 3 & 15 & 0.010 & 18 & 0 & 18 & | \\
\hline & Median (months) & & 60 & 80 & & & 47.5 & 24 & \\
\hline
\end{tabular}

Note: The ESCC cases collected for this study were staged using the NCCN guidelines. 
selection during tumorigenesis. Mutations of SMGs are likely to be 'drivers' in pathogenesis and these genes may affect the biology of a given tumor [7-10]. We and others previously identified SMGs in ESCC patients, including TP53, NOTCH1, PIK3CA, FAT1, CDKN2A, FBXW7, ZNF750, AJUBA, and others [7-10]. We firstly assessed mutational exclusivity among these SMGs as described previously [14]. Most strikingly, an exclusion pattern was observed between the NOTCH1 and PIK3CA mutations in cohort \#1 (Figure 1A). Although one patient was found, and further validated via PCR-Sanger sequencing, with concomitant mutations of NOTCH1 and PIK3CA, the frequency of double mutations was 0.0096 , which is not significantly different from 0.0 by chi-squared analysis. To further test this observation, we extended the exclusivity analysis to cohort \#2 and three other previously published NGS datasets of ESCC patients (cohorts \#3 [7], \#4 [8],

A
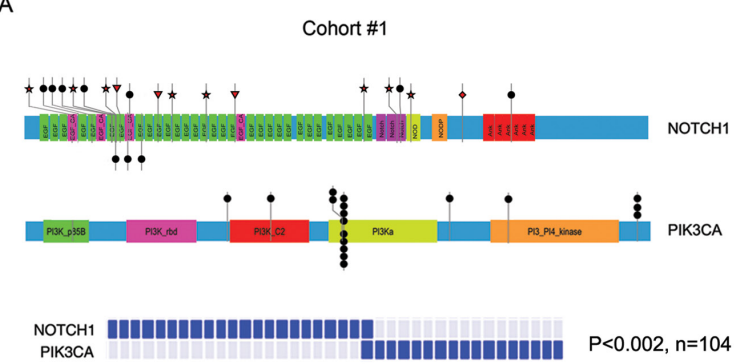

C
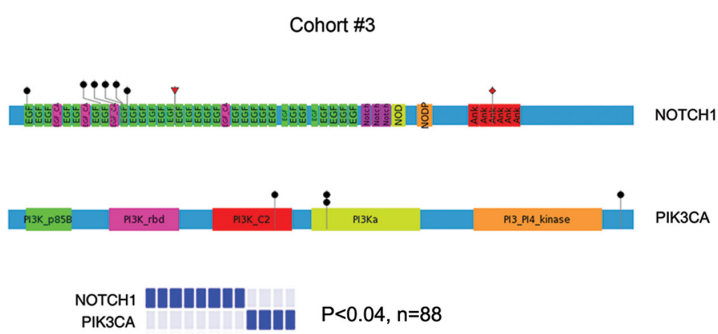

$\mathrm{E}$
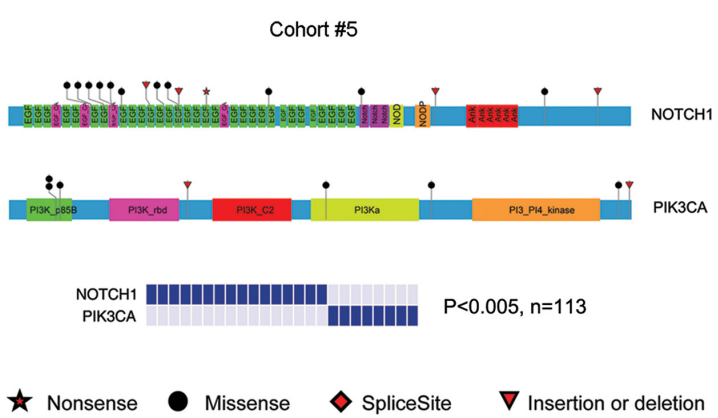

\#5 [9]). Even in this expanded series, mutual exclusivity between the NOTCH1 and PIK3CA mutations was found (Figure 1B-E). When the tumors from cohorts \#1 and \#2 $(\mathrm{n}=193)$, that recruited patients from Taihang Mountains and exhibited similar pattern of NOTCH1 and PIK3CA mutations, were combined, this mutually exclusive pattern between NOTCH1 and PIK3CA mutations was highly significant (Figure 1F). Together, these data suggest that there is a strong inverse relationship between the NOTCH1 and $P I K 3 C A$ mutations in ESCC that was previously undiscovered.

Additionally, the mutated frequency and mutated sites of these two genes were different among ESCC cohorts. The overall frequency of tumor samples with NOTCH1 or PIK3CA mutations in cohort \#1 or \#2 was statistically higher than that of cohort \#3 or \#4 (Supplementary Figure 1B). Moreover, the frequencies
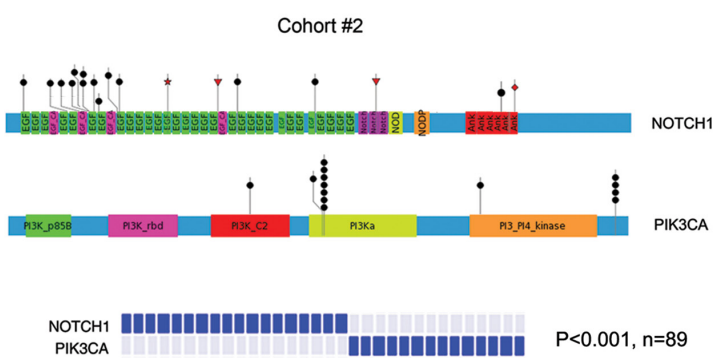

D
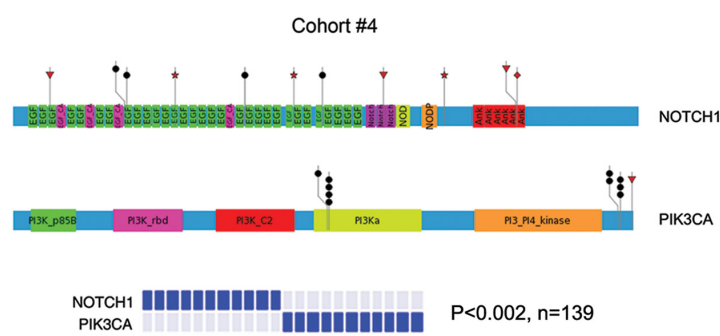

F

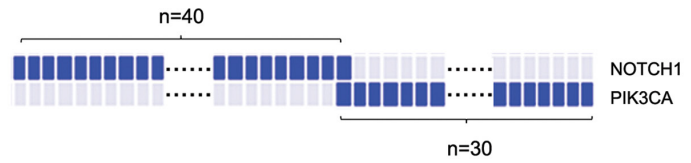

$\mathrm{P}<0.001$, combined cohort $1 \& 2, \mathrm{n}=193$

Figure 1: Exclusion between the mutations of NOTCH1 and PIK3CA in ESCC. The location of somatic mutations on NOTCH1 and PIK3CA (upper panel) and correlation of these mutations (bottom panel) in cohort \#1 A., cohort \#2 B., cohort \#3 C., cohort \#4 D., cohort \#5 E., and combined cohort \#1 and \#2 F. The bottom symbols of NOTCH1 in Figure 1A represent novel mutations that were identified in our analysis according to the sites reported in catalogue of somatic mutations in cancer (COSMIC). The exclusivity of NOTCH1 and PIK3CA mutations was analyzed via modified model proposed by Ewa Szczurek. A $P$ value of $<0.05$ was considered to be statistically significant. 
of the most common tumor-associated PIK3CA mutations, involving either the helical domain (exon 9: c.1624G $>$ A:p.Glu542Lys and c.1633G $>$ A:p.Glu545Lys) or kinase domain (exon 20: c.3140A>G:p.His1047Arg), were significantly different among these cohorts, with $77.8 \%(14 / 18)$ in cohort \#1, 85.7\% (12/14) in cohort $\# 2,75 \%(3 / 4)$ in cohort \#3, and 25\% (2/8) in cohort \#5 (Supplementary Figure 1C).

\section{Clinical and prognostic relevance of $\mathrm{NOTCH} 1$ and $P I K 3 C A$ in ESCC}

Tables 1 summarizes the clinicopathological findings for patients in cohort \#1 that includes 104 ESCC patients, with a median age of 59.68 years old. The estimated median overall survival (OS) was 40.19 months. Seventy patients received the standard chemotherapy (national comprehensive cancer network clinical practice guidelines in oncology, NCCN guidelines). The remaining 34 patients were treated by surgery alone. Additionally, 89 ESCC patients with a median age of 59.1 years and a median OS of 42.66 months were enrolled in cohort \#2. All 89 patients received standard chemotherapy (NCCN guidelines). We then looked for correlations between the NOTCH1 and PIK3CA mutations and clinical parameters. In cohort \#1, NOTCH1 mutations were significantly associated with well-differentiation $(P=0.001)$ and an absence of regional lymph node metastases (N0, $P=$ $0.002)$, and were dramatically enriched in stage I tumors $(P=0.011$, Table 1$)$. We then validated this analysis in cohort \#2. The association of NOTCH1 mutations with tumor stage and lymph node metastasis also held true in this cohort $(P<0.0001$, Supplementary Table 2). Furthermore, the relevance of NOTCH1 mutations to lymph node metastasis was confirmed in our previously
A

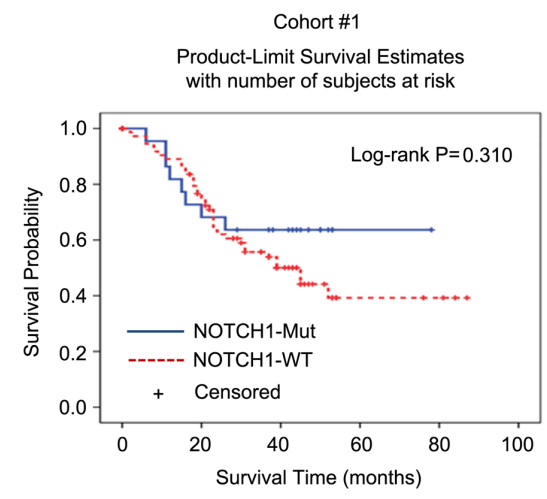

C

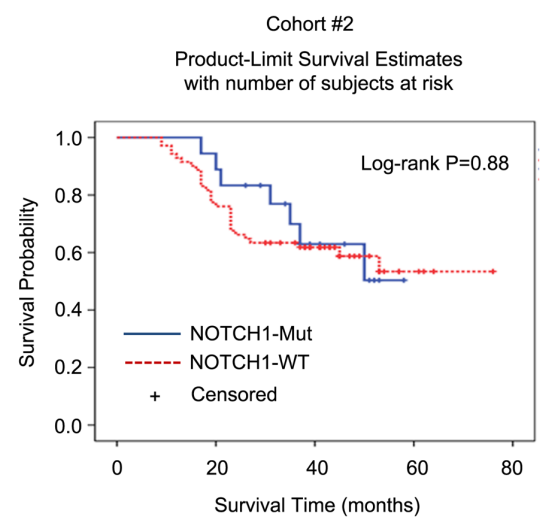

B

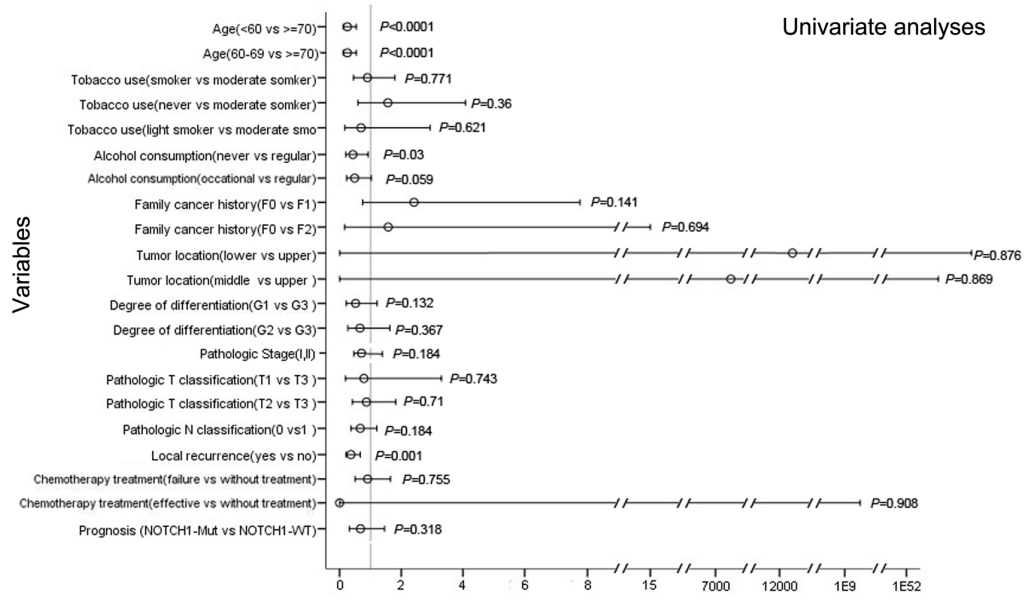

D

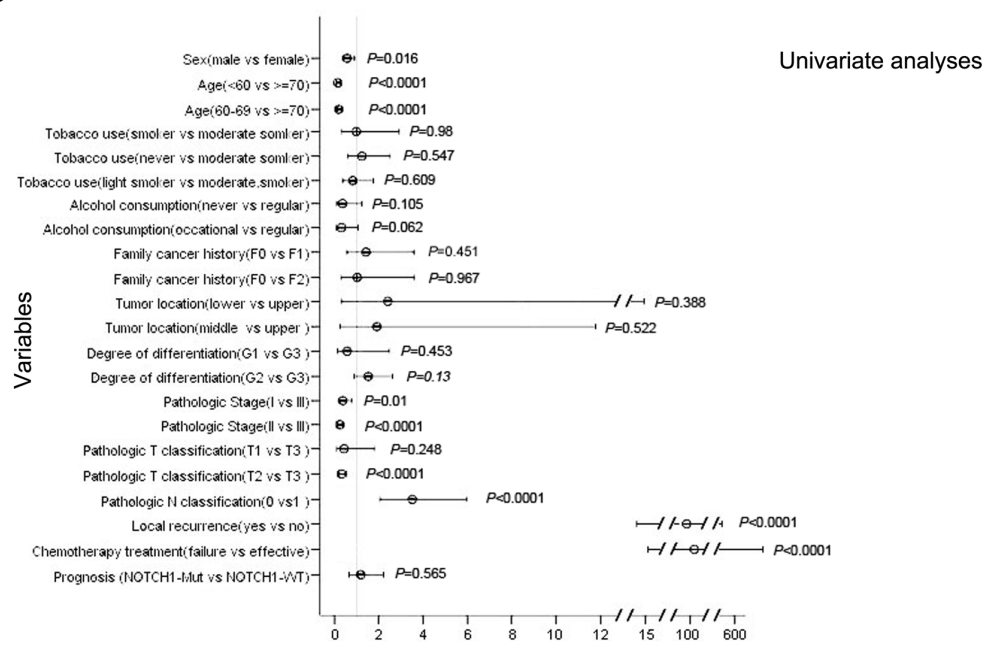

Figure 2: Overall survival among cohorts \#1 and \#2 according to NOTCH1 mutation status. Kaplan-Meier survival curves for patients with NOTCH1 mutations or wild-type in cohort \#1 A. and cohort \#2 C.. The $P$ values were computed by log-rank test. Cox regression analyses were used to adjust for traditional prognostic factors in cohort \#1 B. and cohort \#2 D. . 
published cohort \#3 ( $P=0.011$, Supplementary Table 3) [7]. Collectively, our results strongly suggest that the NOTCH1 mutations were associated with ESCC metastasis; ESCC patients who harbor NOTCH1 mutations show less risk of metastasis. However, $P I K 3 C A$ mutations were not correlated with clinicopathological characteristics, including tumor differentiation, pathologic stage, and lymph node metastasis.

Next, we used Kaplan-Meier analysis to assess the impact of NOTCH1 or PIK3CA mutations on OS in cohort \#1 and validated it in cohort \#2. The patients showed a median OS of 60 months and 80 months for the NOTCH1-mutated and NOTCH1-wild type (WT) groups, respectively, in cohort \#1 (Table 1). Surprisingly, although ESCC patients with NOTCH1 mutations showed less risk of metastasis, the association of NOTCH1 mutations with OS was not statistically significant (Log-rank (MantelCox $), P=0.310$, Figure 2A; Cox regression analysis, $P=$ 0.318 , Figure $2 \mathrm{~B})$. This observation was further validated in cohort \#2 (Cox regression analysis, $P=0.565$, Figure 2D). We did, however, find a significant effect on OS for the PIK3CA mutations. The PIK3CA mutations showed a positive correlation with OS (log-rank $P=0.048$ ) in cohort \#1 (Figure 3A). This trend reached statistical significance in cohort \#2 (Cox regression analysis, $P<0.001$, adjusted hazard ratio $(\mathrm{HR})=0.031,95 \%$ confidence intervals $(\mathrm{CI})$ : 0.006-0.158, Figure 3D) and combined cohort \#1 and \#2 $(\mathrm{P}=0.003$, adjusted HR $=0.241,95 \%$ CI: 0.094-0.622, Supplementary Figure 2E). These data indicate that the PIK3CA mutation may be a marker of favorable prognosis for ESCC patients from the population in Taihang
Mountains, Northern China.

Based on the mutually exclusivity of NOTCH1 and $P I K 3 C A$, ESCC patients could be divided into three groups: patients with NOTCH1 mutations, patients with PIK3CA mutations, and patients without mutations of either gene (Supplementary Table 4). We extended our survival analysis in these groups and found that patients with PIK3CA mutations had a significantly longer OS (median OS of 80.9 months) than patients without mutations in either gene (median OS 40 months) (log-rank $P=0.026$, Figure 4A; Cox regression analysis, $P=0.037$, Figure 4B). Notably, although NOTCH1 mutations were significantly associated with tumor well-differentiation, early pathologic stage, and low lymph node metastasis, patients with NOTCH1 mutations tended to have an even shorter OS than patients with $P I K 3 C A$ mutations; the OS of patients with NOTCH1 mutations was not different from that of patients without mutations in these two genes (Cox regression analysis, $P=0.144$, Figure 4B). A similar trend was observed in cohort \#2 (Figure 4C-D) and combined cohorts \#1 and \#2 (Supplementary Figure 3). PIK3CA mutations correlate with OS (Cox regression analysis, $P<0.001, \mathrm{HR}=0.002$, 95\% CI: 0.0-0.038, Figure 4D), whereas NOTCH1 mutations show no correlation with OS (Cox regression analysis, $P=0.561$, Figure 4D). Apart from NOTCH1 and PIK3CA, no SMGs were statistically associated with clinical variables. Although FAM135B and EP300 were associated with OS in previous studies [7, 9], we did not observe any such statistically significant association in our cohort.
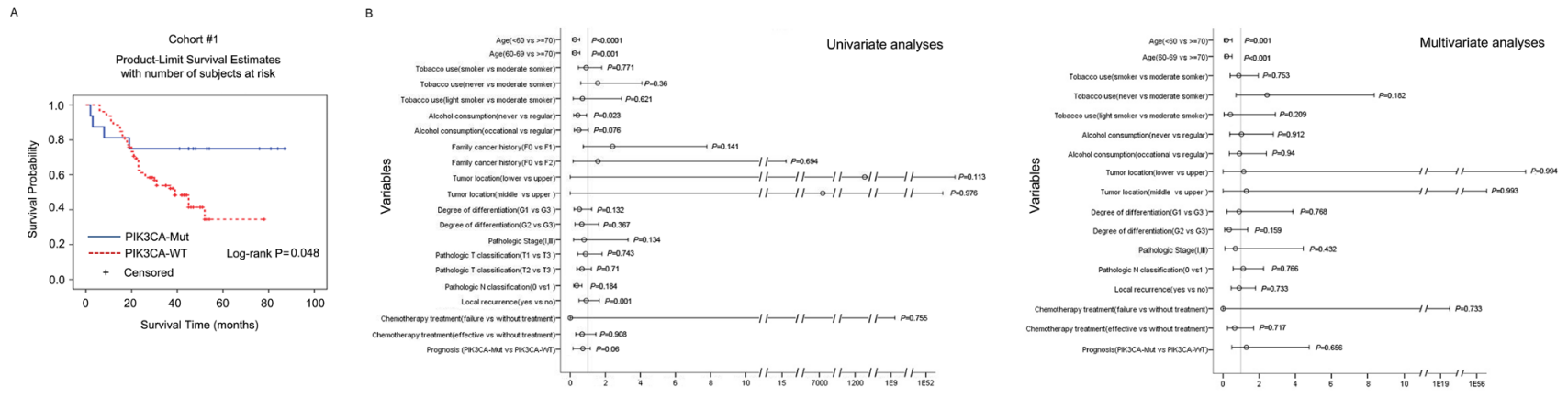

$c$

D
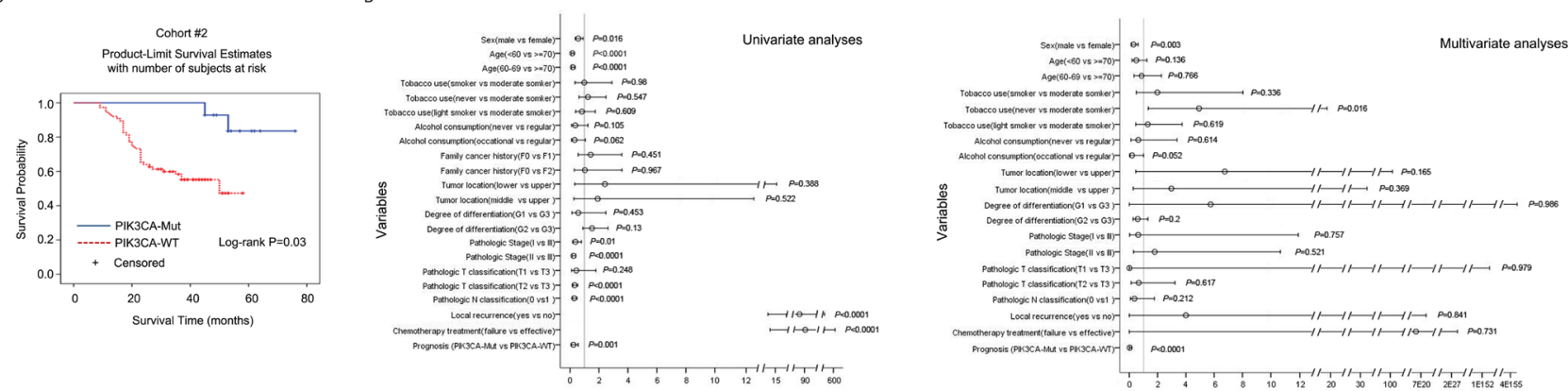

Figure 3: Overall survival among cohorts \#1 and \#2 according to PIK3CA mutation status. Kaplan-Meier survival curves for patients with PIK3CA mutations or wild-type in cohort \#1 A. and cohort \#2 C.. The overall survival data were analyzed using log-rank test, and Cox regression analyses were used to adjust for traditional prognostic factors in cohort \#1 B. and cohort \#2 D. 


\section{Association of $\mathrm{NOTCH} 1$ and $P I K 3 \mathrm{CA}$ mutations with chemotherapy response}

In cohort \#1, 70 patients received standard chemotherapy, including 16 patients with NOTCHI mutations, 12 patients with $P I K 3 C A$ mutations, 1 patient with both the NOTCH1 and PIK3CA mutations, and 41 patients with neither NOTCH1 nor PIK3CA mutations (Supplementary Table 5). Of the 70 patients who received standard chemotherapy, 31 patients responded effectively to chemotherapy; 39 had either tumor progression or tumor recurrence, which were considered to be treatment failures. The median OS of the 70 patients who received standard chemotherapy was 53.48 months. Patients with NOTCH1 mutations showed a median OS of 27.09 months, whereas those with PIK3CA mutations showed a median OS of 80 months. We then evaluated the association between the NOTCH1 and PIK3CA genotype and the efficacy of standard chemotherapy. Of 17 patients who had NOTCH1 mutations and received standard chemotherapy, $70.6 \%$ (12 out of 17) exhibited failure of chemotherapy. Surprisingly, we found no benefit of standard chemotherapy for patients with NOTCHI mutations compared to those with mutations in neither gene $(P=0.389$, Fisher's exact test). Conversely, having a $P I K 3 C A$ mutation was associated with a better response than having mutations in neither gene $(P=0.026$, Fisher's exact test). Of 13 patients with PIK3CA mutations who received standard chemotherapy, only $23 \%$ (3 out of 13 ) showed failure of chemotherapy. Moreover, patients with PIK3CA mutations showed significantly better responses than those with NOTCH1 mutations $(P=0.01$, Fisher's exact test, Figure 5A, right panel).

We validated this trend in cohort \#2, who all received standard chemotherapy treatment. This cohort included 18 patients with NOTCH1 mutations, 14 patients with PIK3CA mutations, and 57 patients mutations in neither gene. Patients in cohort \#2 had a median OS of 44.6 months, with a median OS of 32.78 months for patients with NOTCH1 mutations and 60 months for
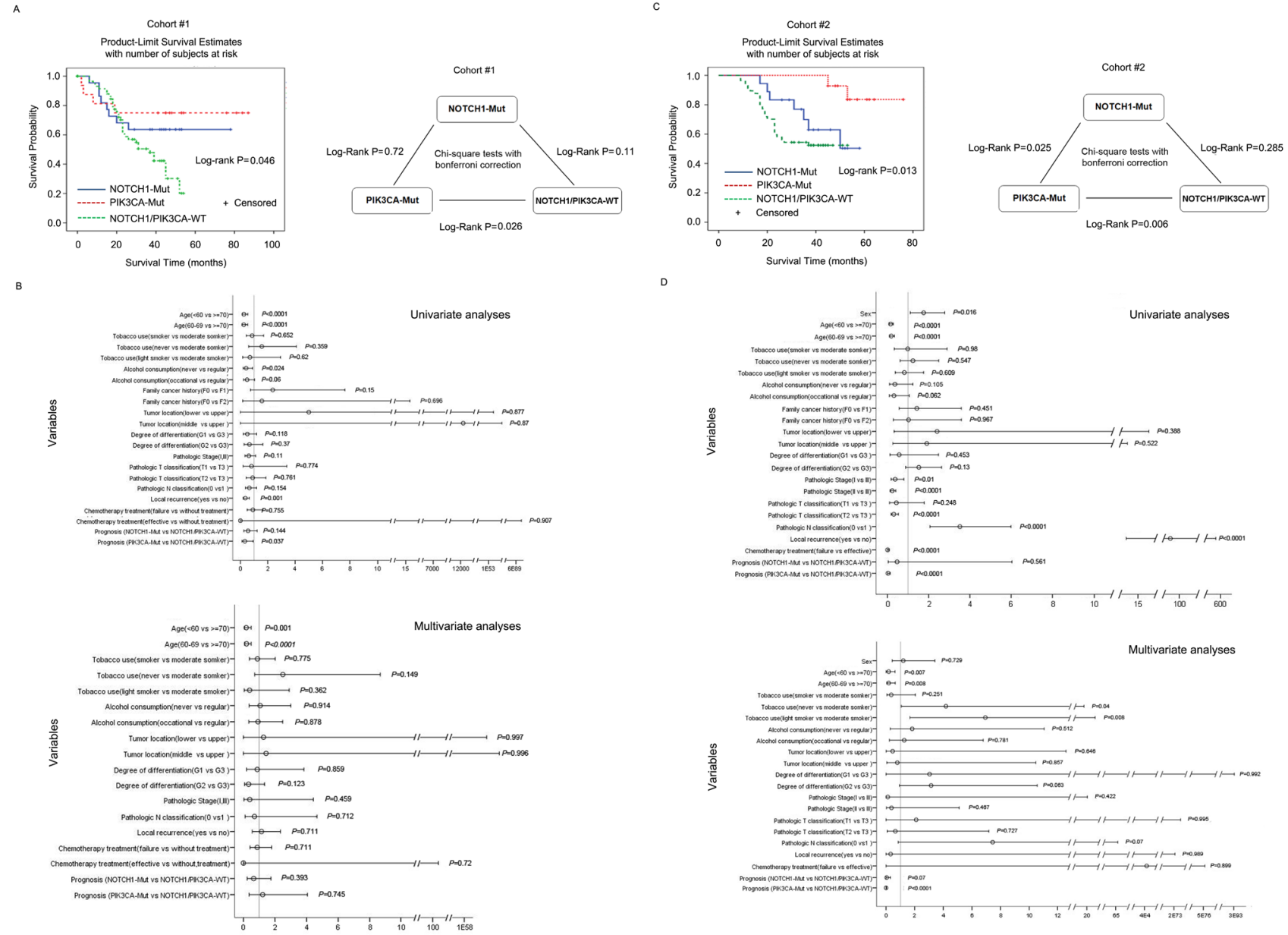

Figure 4: Overall survival among cohorts \#1 and \#2 according to NOTCH1 and PIK3CA mutation status. (A and C) Left panel: Kaplan-Meier survival curves for three groups of patients defined by NOTCH1 and PIK3CA mutations in cohort \#1 A. or cohort \#2 C. The log-rank test was used to calculate the significance. Right panel: chi-square test with Bonferroni correction was used to analyze the OS for the patients in the subgroups. (B and D) Cox regression analysis was used to adjust for traditional prognostic factors in cohort \#1 B. and cohort \#2 D. 
those with PIK3CA mutations. A total of 13 out of 18 patients with NOTCH1 mutations failed to respond to chemotherapy, whereas 12 out of 14 patients with PIK3CA mutations responded to chemotherapy ( $P=0.001$, Fisher's exact test, Supplementary Figure 4A and Supplementary Table 5), consistent with the trend seen in cohort \#1.

Next, we examined the effects of NOTCH1 and PIK3CA mutations on the progression-free survival (PFS) of patients in cohorts \#1 and \#2 who received standard chemotherapy. As expected, patients in cohort \#1 with PIK3CA mutations showed significantly longer PFS than those with NOTCH1 mutations by Log-rank (Mantel-Cox) $(P=0.018$, Figure 5B) and univariate analyses $(P=0.03$, $\mathrm{HR}=4.124,95 \% \mathrm{CI}: 1.145-14.86$, Figure 5C). A similar trend was observed in cohort \#2 (Supplementary Figure 4B-C). Our data suggest that patients with NOTCH1 mutations, who are likely to exhibit a better outcome without chemotherapy, might suffer unnecessarily

A
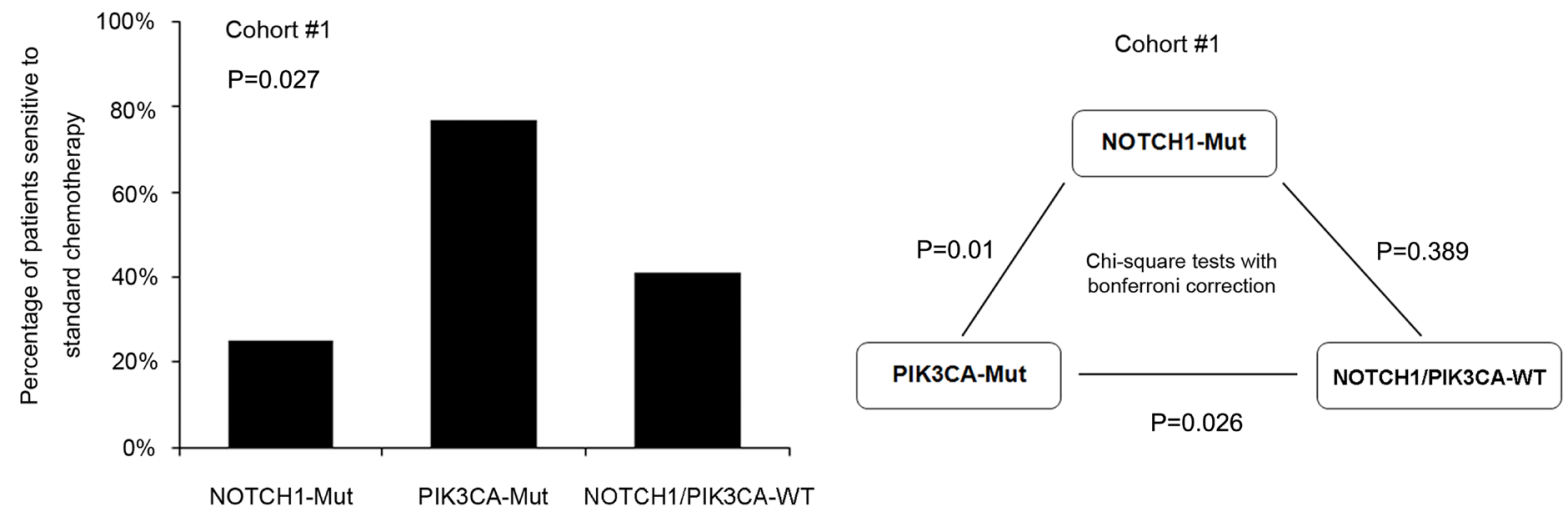

B

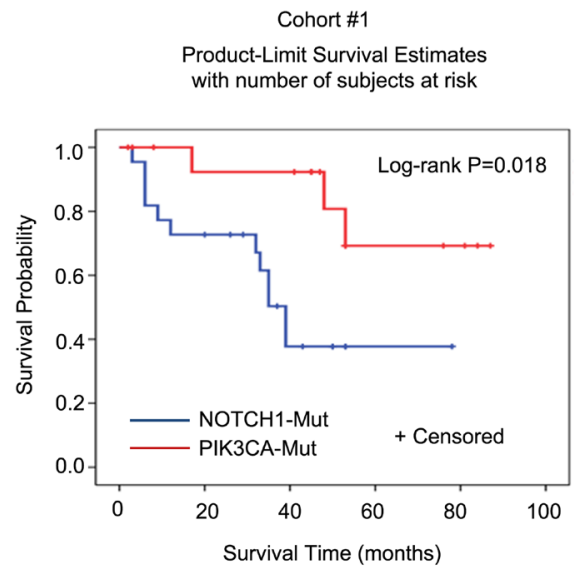

C

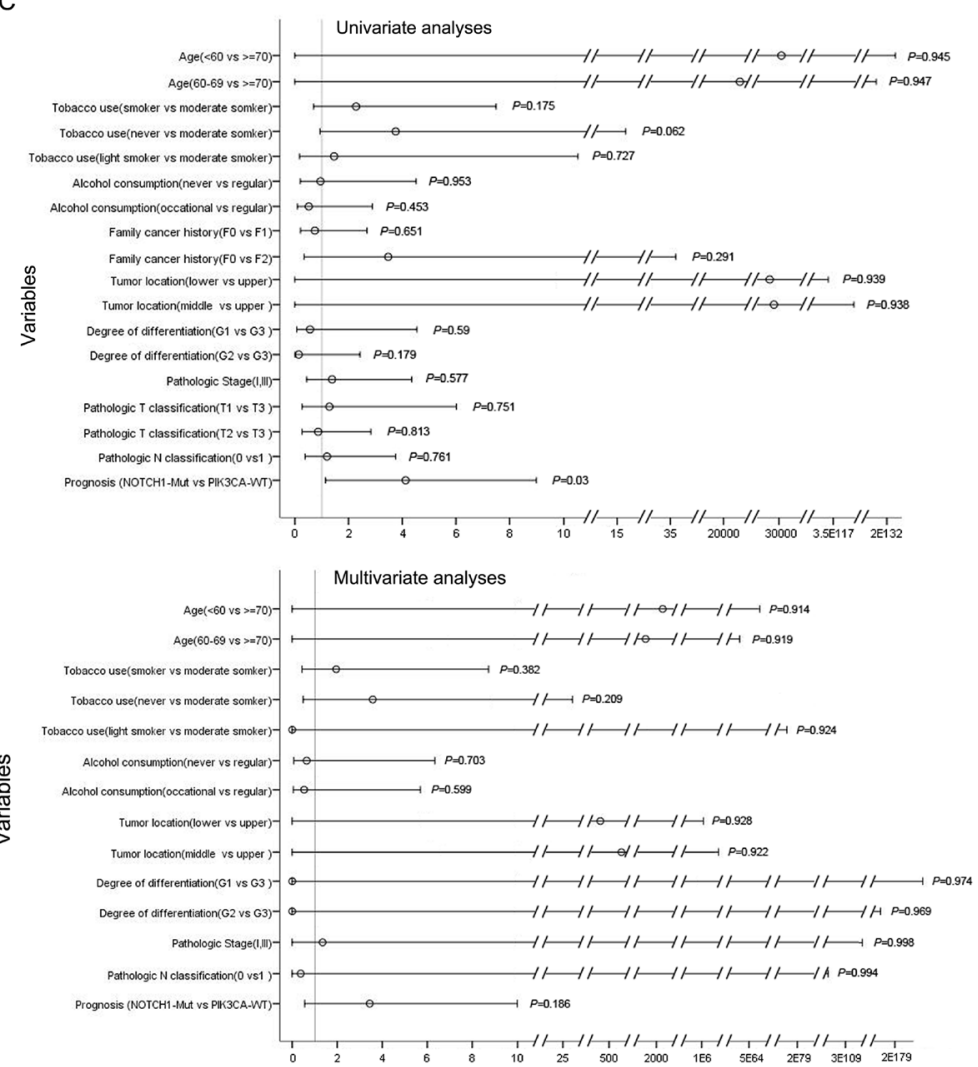

Figure 5: Chemotherapy response and mutation status of NOTCH1 and PIK3CA. A. Left panel: The frequencies of the patients who exhibited sensitivity to standard chemotherapy are described in three groups: patients with NOTCH1 mutations, PIK3CA mutations, or patients with neither, in cohort \#1. Right panel: chi-square test with Bonferroni correction was used to compare the chemotherapeutic efficacy of the subgroups in cohort \#1. B. PFS of 29 patients with NOTCH1 or PIK3CA mutations in cohort \#1. C. Cox regression analysis was used to examine the PFS. 
from side effects of chemotherapy. However, patients with PIK3CA mutations could benefit from standard chemotherapy, and could thus survive longer. Therefore, PIK3CA mutational status may have a potential role as a biomarker for standard chemotherapy and prognosis in ESCC patients from Taihang Mountains, Northern China.

\section{NOTCH1 may act as a tumor suppressor via regulating tumor growth but not metastasis in ESCC}

In general, abnormalities of poor prognostic factors are found in more malignant and advanced cancer cells [1]. However, in our study, we found frequent NOTCHI mutations in early-stage malignancy and less metastasis to regional lymph nodes, but with poor prognosis in ESCC patients. In China, patients with ESCC medically unfit for surgery if the tumor was relapsed. Therefore, we had very rare relapsed tumors to analyze $\mathrm{NOTCH} 1$ mutation status. Alternatively, we performed mutation analysis of NOTCH1 in regional metastatic lymph nodes from 37 of stage III ESCC patients in cohort \#1 and 19 of stage III ESCC patients in cohort \#2. We found none of NOTCH1 mutations in these regional metastatic lymph nodes, further supporting that $\mathrm{NOTCH1}$ mutations associated with early stage and non-lymph node metastasis. Furthermore, we performed functional analysis of NOTCH1-W745X, which located in the epidermal growth factor (EGF) like ligand-binding domain. In light of previous literatures, functional studies have shown that NOTCH1 gene suppress proliferation and promote differentiation of keratinocytes [15]. Similarly, conditional NOTCH1knockout (mice) develop cutaneous epithelial tumors, and transgenic mice expressing a pan-NOTCH1 inhibitor develop cutaneous squamous cell carcinomas [16-17]. The tumor-suppressive role for $\mathrm{NOTCH} 1$ in squamous cells is also supported by recent sequencing studies of related tumor types, such as squamous cell carcinomas of the head and neck, skin, and lung [15,18-19]. Consistently, a tumorsuppressive role for NOTCH1 in ESCC was supported by MTT assay in KYSE150 and KYSE140 cells (Figure 6AB). However, we observed no correlation of $\mathrm{NOTCHI}$ gene with cell migration and invasion as monitored by the iCELLigence RTCA DP system (Figure 6C-D), indicating that NOTCH1 may involve cell proliferation but not migration and invasion in ESCC cells. Together with our genetic observations, these functional data indicate that NOTCH1 may act as a tumor suppressor via regulating tumor growth but not metastasis in ESCC.

\section{DISCUSSION}

A striking finding of this study is the previously unreported, significant mutually exclusive mutational pattern between NOTCHI and PIK3CA in ESCC.
Moreover, our results suggest that patients who harbor NOTCH1 mutations have a statistically significant association with well differentiation, an early stage of malignancy, and less regional lymph node metastasis; however, they exhibited a poor outcome and failure to respond to standard chemotherapy treatment. In contrast, patients who harbor PIK3CA mutations showed a better response to standard chemotherapy and exhibited favorable survival. Recently, the systematic analysis of the Cancer Genome Atlas (TCGA) Pan-Cancer mutation data set across 12 major cancer types revealed 14 mutually exclusive pairs among $127 \mathrm{SMGs}$, including pairs of TP53 and PIK3CA with significant exclusivity in breast adenocarcinoma[12]. However, mutual exclusivity between NOTCH1 and PIK3CA, which was identified as SMGs via genomic analyses of ESCC patients, has not previously been implicated in any type of cancer [7-10, $12]$.

In this study, we observed different patterns of SMGs, especially different distributions of mutation frequencies and mutated sites of NOTCH1 and PIK3CA between ESCC patients from Northern China and those from Southern China. The frequencies of PIK3CA and NOTCH1 mutations in cohorts \#1 and \#2 (from Taihang Mountain, Northern China) were higher than those of cohorts \#3 (from Chaoshan District, Southern China [7]) and cohorts \#4 and \#5 (recruited from CICAMS, where patients came from all over the country and did not have a limited geographic distribution pattern $[8,9])$. The possible reasons for this pattern could be genetic and environmental etiologic factors $[6,12]$. In the high-risk areas of China, there is a strong tendency toward familial aggregation of ESCC, which suggests that genetic susceptibility in combination with exposure to environmental risk factors contributes to the high rates of ESCC in these areas [20]. In addition to ethnicity, other possible etiologic factors such as lifestyle patterns, nutritional deficiencies, and mycotoxin-contaminated foods could play important roles in these high-risk areas [21-24]. Therefore, the different environmental carcinogens between northern and southern China might cause different genetic diversity (e.g., mutational pattern of SMGs) in ESCC individuals from the Taihang Mountains and Chaoshan area.

Patients diagnosed at early stage of cancer progression may exhibit better survival over time [25]. NOTCH1 was mutated in around $20 \%$ out of ESCC patients, and correlated with well differentiation, early TNM stage, and absence of regional lymph node metastases. The close link between NOTCH1 mutation types and clinicopathological features leads us to speculate that ESCC patients with NOTCH1 mutations may have a better prognosis. However, we did not observe such correlation in our patients. Meanwhile, a worse response to standard chemotherapy was observed on patients with NOTCH1 mutations compared to those with PIK3CA mutations. Patient outcomes may correlate with the clinical 
stage of the cancer at diagnosis and the status whether or not patient has received chemotherapy [26]. However, while chemotherapy remains the backbone of current cancer treatment, it is limited by a narrow therapeutic index, significant toxicity leading to severe side effects and frequent acquired resistance [27]. Considering that NOTCH1 mutations associated with early stage and non-lymph node metastasis that was supporting by both genetics alterations and functional study, however, no statistically significant differences in patient outcome and standard chemotherapy benefit were observed in patients contained NOTCH1 mutations, we speculate that there is a possibility that patients harbor NNOTCH1 mutations who should exhibit a better outcome may suffer side-effect of chemotherapy or receive extra treatment. These obstacles call for novel therapeutic approaches for these patients.

Recently, the role of NOTCH signaling in cancers has been reported, with $N O T C H$ signaling having both oncogenic and tumor-suppressive roles, depending on the cellular context [20]. In our and others' cohorts of ESCC patients, many of the missense mutations in the NOTCH1 gene occurred at or near identified important

A

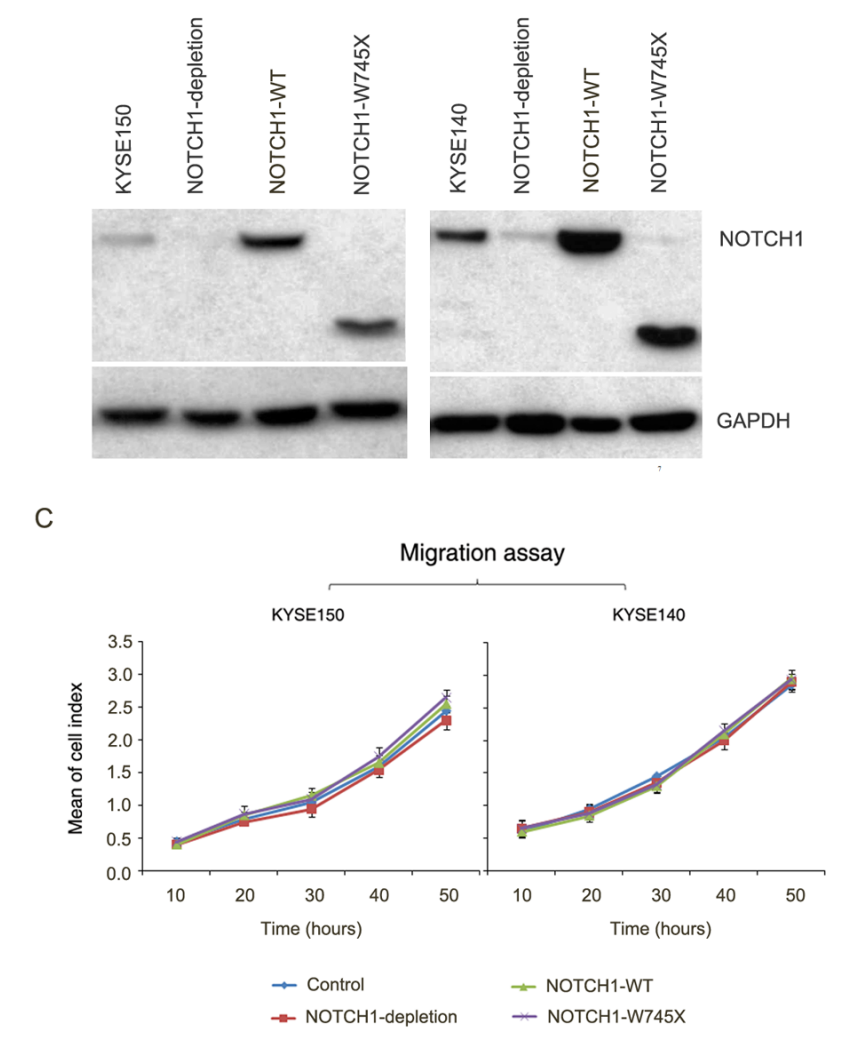

C

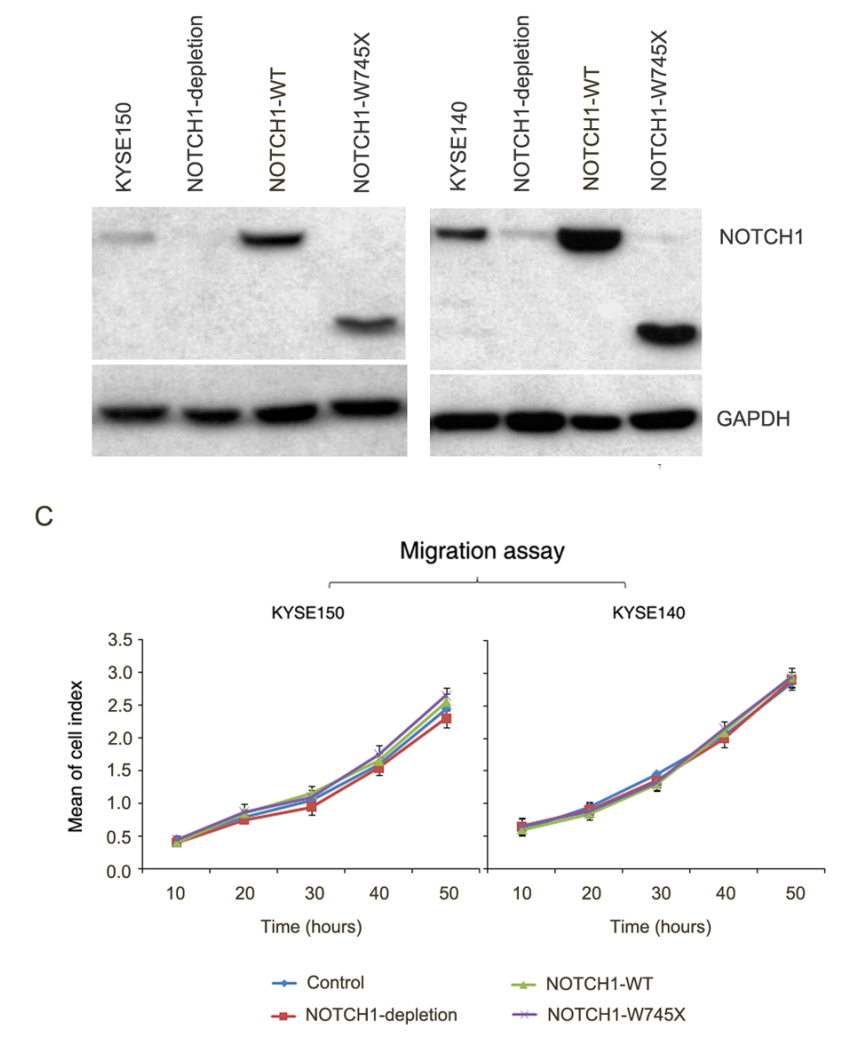

domains, such as the ligand-binding domain (EGF repeats) and the majority of the mutations were predicted to alter the protein N-terminal to the transmembrane region. Moreover, the nonsense mutations (e.g. c.2234G $>$ A: $p$. Trp $745^{*}$ ) observed in NOTCH1 gene generate a premature stop codon, which results in a truncated NOTCH1 protein that lacks the C-terminal domain, which contains a proline-glutamate-serine-threonine (PEST) sequence and is important for transcription activation. Thus, although the activating mutations in NOTCH1 were identified in T-cell acute lymphoblastic leukemia, chronic lymphoblastic leukemia, and breast cancer [21-23], the pattern of the mutations in genes that involve the NOTCH pathway suggests its potential tumor suppressing roles in ESCC, as has been reported for head and neck squamous cell carcinoma, chronic myelomonocytic leukemia, and lung squamous cell carcinoma [20, 24]. This interpretation is consistent with the functional studies of the role of NOTCH1 in ESCC cells, as NOTCH1 depletion promotes tumor cell proliferation in tissue culture. Inhibition of NOTCH1 pathway has been shown to sensitize cancer cells to chemotherapy in prostate cancer, ovarian cancer, colon
B

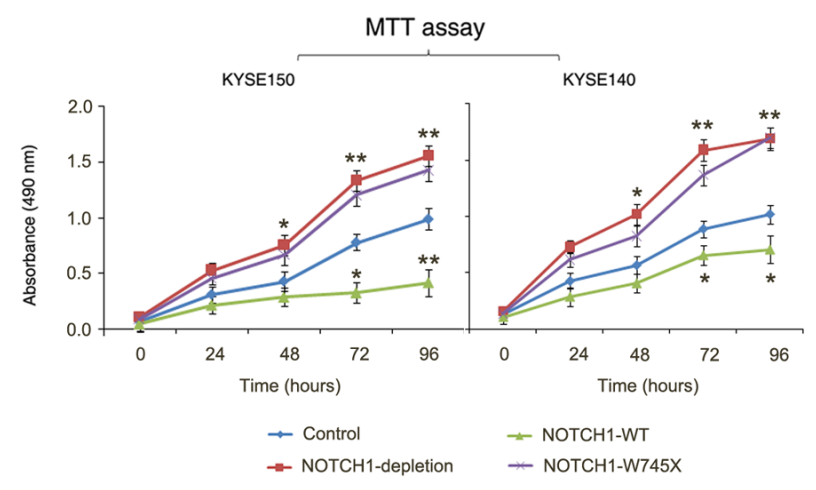

D

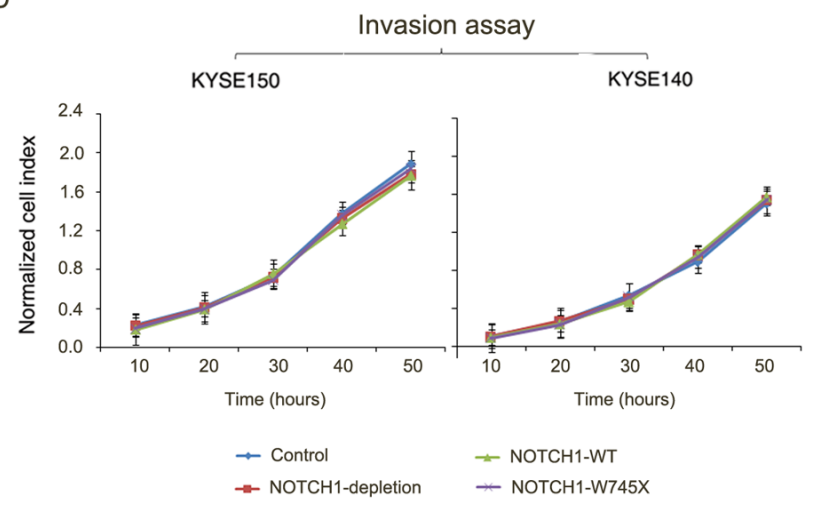

Figure 6: NOTCH1 acts as a tumor suppressor gene in ESCC. A. Knockdown of endogenous NOTCH1 in KYSE150 and KYSE140 cells and subsequent over-expression of NOTCH1 wild-type or W745X mutant were demonstrated by Western Blotting. GAPDH was used as loading control. B. Growth assay shows promoting of proliferation by knockdown NOTCH1 in KYSE150 and KYSE140 cells. Overexpression of NOTCH1 wild-type but not W745X mutant significantly suppressed cell proliferation. Data are mean \pm SD; each experiment was performed in triplicate. ${ }^{* * P}<0.01 ;{ }^{*} P<0.05$. C.-D. Knockdown of NOTCH1 or subsequent over-expression of NOTCH1 wild-type or W745X mutant does not affect migration/invasion of KYSE150 and KYSE140 cells. The data are mean \pm SD; each experiment was performed in triplicate. 
cancer, and glioma [28-31]. Recent studies have aimed to develop antibodies against specific $N O T C H$ receptors and ligands with the hope of limiting side effects while providing the same therapeutic benefit as gamma secretase inhibitors (drugs that inhibit NOTCH signaling); these studies were carried out in human cancers with commonly overactivated mutations in NOTCH1 that confer a survival advantage on the tumors, leading to poorer outcomes for the patients $[15,27]$. However, owing to the potential tumor suppressing roles rather than the oncogenic function of NOTCH1 in ESCC, targeting NOTCH signaling might not be helpful for treating ESCC.

In accordance with the previous literature [32, 34], the PIK3CA mutation could serve as a favorable predictive biomarker in ESCC patients from Taihang Mountains, Northern China. However, PIK3CA mutations were not associated with patient outcomes in ESCC patients from the Chaoshan population, Southern China (cohort \#3) and from CICAMS, which does not have any geographic distribution limitations (cohort \#5) [7,9]. Possible reasons for the conflicting results include the mutation frequency, mutation sites of PIK3CA, or perhaps the sample size of the cohorts. In addition to the different mutation frequencies of PIK3CA among the cohorts, the most common tumor-associated PIK3CA mutations (those involving the helical domain (exon 9: c.1624G>A:p. Glu542Lys and c.1633G>A:p.Glu545Lys) or the kinase domain (exon 20: c.3140A>G:p.His1047Arg)) had significantly different frequencies among these cohorts. In previous studies, the c. $1633 \mathrm{G}>\mathrm{A}$ :p.Glu545Lys and c. $3140 \mathrm{~A}>\mathrm{G}:$ p.His $1047 \mathrm{Arg}$ mutations have been implicated in favorable overall survival in the ESCC patients $[33,34]$. PIK3CA has been named as the key oncogenic effector and could be a potential driver mutation in tumorigenesis of ESCC [35] and these mutants have previously shown oncogenic effects in vivo and may act as a $\mathrm{p}$ otential target site of treatment as in other cancer types[36]. We speculate that the lower frequency of mutations in PIK3CA hotspots in cohort \#5 results in a negative association between PIK3CA mutations and survival. Moreover, our previous mutational signature analysis showed that hotspot mutations (c.1624G $>$ A:p.Glu542Lys, c.1633G $>$ A:p.Glu545Lys) of PIK3CA were significantly enriched in ESCC tumors that had an apolipoprotein B mRNA-editing enzyme catalytic (APOBEC) signature in both cohort \#1 and cohort \#3, which implicates APOBEC activity as a common key driver of PIK3CA mutagenesis in ESCC patients from Northern or Southern populations in China [10]. Therefore, the effect of PIK3CA mutations on survival prognosis might be observed when cohort \#3 is extended to increase the sample size.

Together with previously reported NGS data, our results document for the first time that NOTCHI and $P I K 3 C A$ mutations are mutually exclusive alterations in ESCC. Although NOTCH1 mutations had a statistically significant association with well-differentiation, early stage of malignancy and less regional lymph node metastasis in ESCC, patients who harbor NOTCH1 mutations would not benefit from standard chemotherapy, and alternative therapeutic strategy must be developed for these patients. Conversely, patients who harbor PIK3CA mutations could benefit from standard chemotherapy, and thus PIK3CA mutational status may have a potential role as a biomarker for standard chemotherapy and prognosis. These results raise the possibility for the categorization of ESCC using the mutations of NOTCH1 and PIK3CA. However, the NOTCH1 and PIK3CA mutated samples represented a small proportion of ESCC, and the majority of samples had wild-type NOTCH1 and PIK3CA. Further studies of larger patient cohorts including all stages ESCC from multiple restricted areas with high incidences of ESCC will be necessary to validate the mutual exclusivity of NOTCH1 and PIK3CA and the relationship of these two genes with clinical features, as well as to further refine the potential subclassification of ESCC based on these genetic alterations. Whether they indeed reflect the biology of ESCC and the patients' clinical course remains to be seen. The results could extend our knowledge to potential molecular diagnosis markers, predicting overall patient survival and chemotherapy response in a portion of ESCC patients, which could guide the therapeutic strategy to combat ESCC.

\section{MATERIALS AND METHODS}

\section{Ethics approval}

This study was approved by the ethical committee of the Shanxi and Henan, China (IRB of Shanxi Medical University, Approval No. 2009029, and the Ethics Committee of Henan Cancer Hospital, Approval No. 2009xjs 12). All samples were obtained before treatment according to the guidelines of the local ethical committees, and written informed consent was received from all participants prior to inclusion in the study.

\section{Patient characteristics}

A total of 104 ESCC patients from the Taihang Mountains were recruited as described previously [10]. Pairs of tumors and matched normal tissues from 14 patients underwent whole-genome sequencing (WGS, median coverage of $65 \times$ ), and 90 pairs of samples underwent whole-exome sequencing (WES, median coverage of $132 \times$ ) [10]. Additionally, to provide highconfidence mutations, 96 pairs of tumors and matched normal tissue were selected for deep target capture-based validation (TCS, at least $365 \times$ ). A detailed description of next-generation sequencing analysis pipeline is provided in the Online Methods. All subsequent analyses relied 
on these validated data and not on the primary genome discovery sequence. A summary of clinical characteristics of the analyzed patients (cohort \#1) is presented in Table 1 and Supplementary Table 1. In this cohort, seventy patients received standard chemotherapy (NCCN guidelines). Most of the patients had undergone platinum based chemotherary conbined with 5-fluorouracil (FU) or paclitaxel. Patients were treated with paclitaxel on the first day combined with platinum on day 1 or devided into 4-5 days. 5-FU was used from day 1 to day 5 or continuous infusion combined with platinum as above. A small part of patients used concomitant chemotherapy was continuous infusion of $5-\mathrm{FU}$ alone with a dose of $300 \mathrm{mg} / \mathrm{m} 2 /$ day during the whole course of treatment . Treatment cycles were repeated every 3-4 weeks (Supplementary Table 5). Overall survival (OS) data were available for 95 patients. We examined the prevalence of overall failure of standard chemotherapy in cohort \#1, with treatment failure as either occurrence of cancer-related death or emergence of recurrent tumors in patients who received standard chemotherapy.

We further validated our results in an extended ESCC cohort (cohort \#2). A total of 89 ESCC patients from Shanxi and Henan provinces, Taihang Mountains received standard chemotherapy ( $\mathrm{NCCN}$ guidelines) and were randomly assigned to cohort \#2 (Supplementary Table 1-2). The genotype of NOTCH1 and PIK3CA was analyzed in 89 formalin-fixed paraffin-embedded tumors by PCR-Sanger sequencing using the paired primers $1 \mathrm{~F}$ (ACCCGATGCGGTTAGAGCC), $1 \mathrm{R}$ (ACACAATAGTGTCTGTGACTCC), 2F (ACTCCATGCTTAGAGTTGGAG), and 2R (GGATTGTGCAATTCCTATGCAATC) for PIK3CA and $1 \mathrm{~F}$ (GTGACTGCTCCCTCAACTTCAAT), 1R (CTGTCACAGTGGCCGTCACT), 2F (GTCAACGCCGTAGATGACCT), and 2R (TCTCCTCCCTGTTGTTCTGC) for NOTCH1. Moreover, we validated our result in another three independent ESCC mutation datasets: Cohort \#3 from our previous report of 17 WGS and 71 WES samples, which was recruited from the Chaoshan District of Guangdong Province, another area that has high ESCC prevalence in China [7]. Cohort \#4 was from Lin et al. of 20 WES and 119 TCS samples, which were recruited from CICAMS and Linxian Cancer Hospital [8]. Cohort \#5 from Gao et al. contained 113 WES samples, which were recruited from CICAMS [9].

\section{Mutually exclusive pattern}

The mutual exclusivity pattern was detected as described previously [14]. Briefly, n columns correspond to NOTCH1 and PIK3CA mutations and $\mathrm{m}$ rows correspond to patients whose tumor samples were collected (with $\mathrm{m} \mathrm{n}$ ). For each patient and gene, the dataset records a binary alteration status of the gene observed in the patient, with 0 standing for absence and 1 for presence of alteration. We define the set of model parameters $\theta=\{\gamma, \delta, \alpha, \beta\}$ with coverage $\gamma$, impurity $\delta$, false positive rate $\alpha$ and false negative rate $\beta$; and a set of random variables including variable $\mathrm{C}$ that indicates patient coverage, variable $\mathrm{H}$ that specifies the single exclusively mutated gene in a covered patient, variable $\mathrm{T}$ that represent the true alterations of genes, and variable $\mathrm{Y}$ that correspond to the alteration status of genes recorded in the data. The model is defined by: $P\left(Y_{g} \mid T_{g}, \theta\right)=\alpha^{Y_{g}\left(1-T_{g}\right)}(1-\alpha)^{\left(1-Y_{g}\right)\left(1-T_{g}\right)} \beta^{\left(1-Y_{g}\right) T_{g}}(1-\beta)^{Y_{g} T_{g}}$. . A P value of $<0.05$ was considered to be statistically significant.

\section{Statistical analyses}

Fisher's exact test was used to analyze categorical features such as the association of NOTCH1 or PIK3CA mutations with clinical and pathological features, the distribution of gene mutations in different clusters or different cohorts, and the response rate to chemotherapy among the subgroups. A $P$ value of $<0.05$ was considered to be statistically significant. Comparisons of binary and categorical patient characteristics between subgroups were performed using the chi-square test with Bonferroni correction. A $P$ value of $<0.025$ was considered to be statistically significant.

The OS and progression-free survival (PFS) distributions were described by the Kaplan-Meier curves, and statistical significance was calculated using the logrank test. OS was evaluated from the time of diagnosis to death or the final follow-up. Censored cases were defined as patients who lost contact during the follow-up and who were still alive at the end of the study. PFS was evaluated from the time of diagnosis to the occurrence of cancerrelated death or emergence of recurrent tumors or the last follow-up. Censored cases were defined as patients who were still alive and had no recurrent tumors at the end of the study. The primary outcome was clinical benefit, which was defined as failure or response to chemotherapy. The occurrence of cancer-related death or the emergence of recurrent tumors in patients who had received standard chemotherapy was considered to represent failure of chemotherapy. Univariate and multivariate analyses with the Cox proportional hazards model were used to examine the association between mutations and overall survival and response to chemotherapy. For multivariate analysis, in addition to the confounders with univariate $P$ values of $<0.05$, traditional prognostic factors, including age, smoking, drinking, clinical stage, tumor differentiation, and chemotherapy treatment, were adjusted for the analysis of the prognosis. 


\section{NOTCH1 knockdown and its mutant over- expression}

Lentivirus vector pLKO.1-puro and its packaging plasmids pMD2.G and psPAX2 were obtained from Addgene. NOTCH1 knockdown experiments were performed in two ESCC lines with high endogenous NOTCH1 expression: KYSE150 and KYSE140. Two independent shRNAs, (5'-UCGCAUUGACCAUUCAAACUGGUGG-3', Scrambled siRNA 5'-AAAUGUGUGUACGUCUCCUCC-3'; NOTCH1 or nontargeting ON-TARGET plus SMART siRNAs, Dharmacon RNATechnologies), were cloned into pLKO.1puro vector as described previously [10]. Infection of retrovirus containing both siRNAs resulted in similar phenotype changes in the pre-experiments (data not shown). Thus, in order to obtain high infection efficiency, we pooled these two independent siRNAs retrovirus to infect KYSE cells. A non-specific targeting shRNA was also cloned into pLKO.1-puro vector using as scrambled control. Relative expression of $\mathrm{NOTCH} 1$ was normalized to GAPDH expression level. For overexpression experiments, $\mathrm{NOTCH} 1$ wild-type and $\mathrm{NOTCH} 1$-W745X mutant were cloned into pLV-EGFP(2A)-puro-GFPvector and validated by sequencing. For overexpression experiments, we used pLV-EGFP(2A)-puro-GFP-vector as a control. NOTCH1-depleted KYSE cells were infected with viruses as previously described [10]. 24 hours post infection, cells were subjected for subsequent experiments.

\section{Immunoblotting}

Cells were lysed for $30 \mathrm{~min}$ in Triton buffer (1\% Triton X-100, $50 \mathrm{mM}$ Tris- $\mathrm{HCl} \mathrm{pH}$ 7.6, $150 \mathrm{mM} \mathrm{NaCl}$, $1 \%$ sodium deoxycholate, $0.1 \%$ SDS) supplemented with protease and phosphatase inhibitors $(1 \mathrm{mM}$ PMSF, $2 \mathrm{mM}$ sodium pyrophosphate, $2 \mathrm{mM}$ sodium betaglycerophosphate, $1 \mathrm{mM}$ sodium fluoride, $1 \mathrm{mM}$ sodium orthovanadate, $10 \mu \mathrm{g} / \mathrm{mL}$ leupeptin and $10 \mu \mathrm{g} /$ $\mathrm{mL}$ aprotinin). Lysates were cleared by centrifugation at $15,000 \mathrm{x}$ g at $4{ }^{\circ} \mathrm{C}$ for $15 \mathrm{~min}$, and protein concentrations were determined using the Bradford method. Fifty $\mu \mathrm{g}$ of protein were separated by SDS-polyacrylamide gel electrophoresis and transferred onto Immobilon-P membranes. Proteins were detected by using anti-Nterminal NOTCH1 antibody (GENWAY Biotech Inc). Antibody binding was detected using horseradish peroxidase-labelled anti-mouse (Sigma) or anti-rabbit (Cell Signaling) antibodies and chemiluminescence was detected using a LAS4000 device (Fuji). Equal protein loading was confirmed with antibodies against GAPDH (Transgen).

\section{MTT assay}

$4 \times 10^{3}$ cells were seeded in 96-well plates and incubated in normal condition for 24 hours. Cells were treated with $100 \mu \mathrm{l}$ of $5 \mathrm{mg} / \mathrm{ml}$ of MTT (Invitrogen) solution for 4 hours at $37^{\circ} \mathrm{C}$ until crystals were formed. MTT solution was removed from each well and $100 \mu \mathrm{l}$ of DMSO was added to each well to dissolve the crystals. Color intensity was measured by Microplate Reader (Bio-Rad) at $490 \mathrm{~nm}$. Each experiment consisted of four replications and at least three independent experiments were carried out.

\section{Migration/invasion assay}

Migration and invasion assays were performed in 16-well CIM plates in an xCELLigence RTCA DP system (ACEA Biosciences) using matrigel basement membrane matrix (BD) for real-time cell migration analysis as described previously [10]. Briefly, 30,000 cells per well were seeded as 5 duplicates in serum-free medium at the upper compartment of the CIM plates coated with or without matrigel. Serum-complemented medium was added to the lower compartment of the chamber, and then started measurement in xCELLigence RTCA DP system and analyzed the CI (Cell Index) curves to determine cell invasion activity. For negative controls, we added serumfree medium at both upper and bottom chambers. The concentration of matrigel was 1:6 for KYSE150 cells and 1:10 for KYSE140 cells. The cell index representing the amount of migrated cells was calculated with the RTCA Software from ACEA Biosciences (San Diego, CA). At least three independent experiments were carried out; for each independent experiment, 5 duplicates were performed for each group.

\section{GRANT SUPPORT}

This work was supported by funding from the National Natural Science Foundation of China (81330063 and 81272189 to Y.C., 81021061 and 81230047 to Q.M.Z., 81272694 to X. C, 81201956 to J.L., 81402342 to L.Z., and 81502135 to P.K.), the Key Project of Chinese Ministry of Education (NO213005A to Y.C.), the Specialized Research Fund for the Doctoral Program of Higher Education (20121417110001 to Y.C.), a research project supported by Shanxi Scholarship Council of China (2013-053 to Y.C.), the Innovative Team in Science \& Technology of Shanxi (2013131023 to Y.C.), and the Program for the Outstanding Innovative Teams of Higher Learning Institutions of Shanxi (to Y.C.).

\section{CONFLICTS OF INTEREST}

The authors declare that they have no competing 
financial interests.

\section{REFERENCES}

1. Torre LA, Bray F, Siegel RL, Ferlay J, Lortet-Tieulent JJemal A. Global cancer statistics, 2012. CA: a cancer journal for clinicians. 2015; 65:87-108.

2. Pennathur A, Gibson MK, Jobe BALuketich JD. Oesophageal carcinoma. Lancet. 2013; 381:400-412.

3. Sakaeda T, Yamamori M, Kuwahara ANishiguchi K. Pharmacokinetics and pharmacogenomics in esophageal cancer chemoradiotherapy. Advanced drug delivery reviews. 2009; 61:388-401.

4. Perez-Herrero EFernandez-Medarde A. Advanced targeted therapies in cancer: Drug nanocarriers, the future of chemotherapy. European journal of pharmaceutics and biopharmaceutics. 2015; 93:52-79.

5. Li XY, Su M, Huang HH, Li H, Tian DPGao YX. mtDNA evidence: genetic background associated with related populations at high risk for esophageal cancer between Chaoshan and Taihang Mountain areas in China. Genomics. 2007; 90:474-481.

6. Wu C, Wang Z, Song X, Feng XS, Abnet CC, He J, Hu N, Zuo XB, Tan W, Zhan Q, Hu Z, He Z, Jia W, et al. Joint analysis of three genome-wide association studies of esophageal squamous cell carcinoma in Chinese populations. Nature genetics. 2014; 46:1001-1006.

7. Song Y, Li L, Ou Y, Gao Z, Li E, Li X, Zhang W, Wang J, Xu L, Zhou Y, Ma X, Liu L, Zhao Z, et al. Identification of genomic alterations in oesophageal squamous cell cancer. Nature. 2014; 509:91-95.

8. Lin DC, Hao JJ, Nagata Y, Xu L, Shang L, Meng X, Sato Y, Okuno Y, Varela AM, Ding LW, Garg M, Liu LZ, Yang $\mathrm{H}$, et al. Genomic and molecular characterization of esophageal squamous cell carcinoma. Nature genetics. 2014; 46:467-473.

9. Gao YB, Chen ZL, Li JG, Hu XD, Shi XJ, Sun ZM, Zhang F, Zhao ZR, Li ZT, Liu ZY, Zhao YD, Sun J, Zhou CC, et al. Genetic landscape of esophageal squamous cell carcinoma. Nature genetics. 2014; 46:1097-1102.

10. Zhang L, Zhou Y, Cheng C, Cui H, Cheng L, Kong P, Wang J, Li Y, Chen W, Song B, Wang F, Jia Z, Li L, et al. Genomic analyses reveal mutational signatures and frequently altered genes in esophageal squamous cell carcinoma. American journal of human genetics. 2015; 96:597-611.

11. Cohen AL, Piccolo SR, Cheng L, Soldi R, Han B, Johnson WEBild AH. Genomic pathway analysis reveals that EZH2 and HDAC4 represent mutually exclusive epigenetic pathways across human cancers. BMC medical genomics. 2013; 6:35.

12. Kandoth C, McLellan MD, Vandin F, Ye K, Niu B, Lu C, Xie M, Zhang Q, McMichael JF, Wyczalkowski MA, Leiserson MD, Miller CA, Welch JS, et al. Mutational landscape and significance across 12 major cancer types. Nature. 2013; 502:333-339.

13. Kiel MJ, Velusamy T, Rolland D, Sahasrabuddhe AA, Chung F, Bailey NG, Schrader A, Li B, Li JZ, Ozel AB, Betz BL, Miranda RN, Medeiros LJ, et al. Integrated genomic sequencing reveals mutational landscape of T-cell prolymphocytic leukemia. Blood. 2014; 124:1460-1472.

14. Szczurek EBeerenwinkel N. Modeling mutual exclusivity of cancer mutations. PLoS Comput Biol. 2014; 10:e1003503.

15. Dotto GP. Notch tumor suppressor function. Oncogene. 2008 Sep 1;27:5115-5123.

16. Nicolas M, Wolfer A, Raj K, Kummer JA, Mill P, van Noort M, Hui CC, Clevers H, Dotto GP, Radtke F. Notch1 functions as a tumor suppressor in mouse skin. Nat Genet. 2003 Mar;33:416-421.

17. Proweller A, Tu L, Lepore JJ, Cheng L, Lu MM, Seykora J, Millar SE, Pear WS, Parmacek MS. Impaired notch signaling promotes de novo squamous cell carcinoma formation. Cancer Res. 2006 Aug 1;66:7438-7444.

18. Stransky N, Egloff AM, Tward AD, Kostic AD, Cibulskis K, Sivachenko A, Kryukov GV, Lawrence MS, Sougnez C, McKenna A, Shefler E, Ramos AH, Stojanov P, et al. The mutational landscape of head and neck squamous cell carcinoma. Science. 2011 Aug 26;333:1157-1160.

19. Wang NJ, Sanborn Z, Arnett KL, Bayston LJ, Liao W, Proby CM, Leigh IM, Collisson EA, Gordon PB, Jakkula L, Pennypacker S, Zou Y, Sharma M,et al. Loss-offunction mutations in Notch receptors in cutaneous and lung squamous cell carcinoma. Proc Natl Acad Sci U S A. 2011 Oct 25;108:17761-17766.

20. Agrawal N, Frederick MJ, Pickering CR, Bettegowda C, Chang K, Li RJ, Fakhry C, Xie TX, Zhang J, Wang J, Zhang N, El-Naggar AK, Jasser SA, et al. Exome sequencing of head and neck squamous cell carcinoma reveals inactivating mutations in NOTCH1. Science. 2011; 333:1154-1157.

21. Mittal S, Subramanyam D, Dey D, Kumar RVRangarajan A. Cooperation of Notch and Ras/MAPK signaling pathways in human breast carcinogenesis. Molecular cancer. 2009; $8: 128$.

22. Witkowski MT, Cimmino L, Hu Y, Trimarchi T, Tagoh H, McKenzie MD, Best SA, Tuohey L, Willson TA, Nutt SL, Busslinger M, Aifantis I, Smyth GK, et al. Activated Notch counteracts Ikaros tumor suppression in mouse and human T-cell acute lymphoblastic leukemia. Leukemia. 2015; 29:1301-1311.

23. Fabbri G, Rasi S, Rossi D, Trifonov V, Khiabanian H, Ma J, Grunn A, Fangazio M, Capello D, Monti S, Cresta S, Gargiulo E, Forconi F, et al. Analysis of the chronic lymphocytic leukemia coding genome: role of NOTCH1 mutational activation. The Journal of experimental medicine. 2011; 208:1389-1401.

24. Klinakis A, Lobry C, Abdel-Wahab O, Oh P, Haeno H, Buonamici S, van De Walle I, Cathelin S, Trimarchi T, Araldi E, Liu C, Ibrahim S, Beran M, et al. A novel tumour- 
suppressor function for the Notch pathway in myeloid leukaemia. Nature. 2011; 473:230-233.

25. Sturm D, Witt H, Hovestadt V, Khuong-Quang DA, Jones DT, Konermann C, Pfaff E, Tönjes M, Sill M, Bender S, Kool M, Zapatka M, Becker N, et al. Hotspot mutations in H3F3A and IDH1 define distinct epigenetic and biological subgroups of glioblastoma. Cancer Cell 2012;22:425-437.

26. Zheng Y, Cao X, Wen J, Yang H, Luo K, Liu Q, Huang Q, Chen J, Fu J. Smoking affects treatment outcome in patients with resected esophageal squamous cell carcinoma who received chemotherapy. PLoS One 2015;10:e0123246.

27. Pavelic J. Editorial: combined cancer therapy. Curr Pharm Des 2014;20:6511-6512.

28. Ye QF, Zhang YC, Peng XQ, Long Z, Ming YZHe LY. siRNA-mediated silencing of Notch-1 enhances docetaxel induced mitotic arrest and apoptosis in prostate cancer cells. Asian Pacific journal of cancer prevention : APJCP. 2012; 13:2485-2489.

29. McAuliffe SM, Morgan SL, Wyant GA, Tran LT, Muto KW, Chen YS, Chin KT, Partridge JC, Poole BB, Cheng KH, Daggett J, Jr., Cullen K, Kantoff E, et al. Targeting Notch, a key pathway for ovarian cancer stem cells, sensitizes tumors to platinum therapy. Proceedings of the National Academy of Sciences of the United States of America. 2012; 109:E2939-2948.

30. Meng RD, Shelton CC, Li YM, Qin LX, Notterman D, Paty PBSchwartz GK. gamma-Secretase inhibitors abrogate oxaliplatin-induced activation of the Notch-1 signaling pathway in colon cancer cells resulting in enhanced chemosensitivity. Cancer research. 2009; 69:573-582.

31. Wang J, Wakeman TP, Lathia JD, Hjelmeland AB, Wang XF, White RR, Rich JNSullenger BA. Notch promotes radioresistance of glioma stem cells. Stem cells. 2010; 28:17-28.

32. Takebe N, Nguyen DYang SX. Targeting notch signaling pathway in cancer: clinical development advances and challenges. Pharmacology \& therapeutics. 2014; 141:140149.

33. Shigaki H, Baba Y, Watanabe M, Murata A, Ishimoto T, Iwatsuki M, Iwagami S, Nosho KBaba H. PIK3CA mutation is associated with a favorable prognosis among patients with curatively resected esophageal squamous cell carcinoma. Clinical cancer research. 2013; 19:2451-2459.

34. Hou J, Jiang D, Zhang J, Gavine PR, Xu S, Liu Y, Xu C, Huang J, Tan Y, Wang H, Lu Y, Zheng L, Hou Y, et al. Frequency, characterization, and prognostic analysis of PIK3CA gene mutations in Chinese esophageal squamous cell carcinoma. Human pathology. 2014; 45:352-358.

35. Murugan AK, Munirajan AKTsuchida N. Genetic deregulation of the PIK3CA oncogene in oral cancer. Cancer letters. 2013; 338:193-203.

36. Janku F, Wheler JJ, Naing A, Stepanek VM, Falchook GS, Fu S, Garrido-Laguna I, Tsimberidou AM, PihaPaul SA, Moulder SL, Lee JJ, Luthra R, Hong DS, et al.
PIK3CA mutations in advanced cancers: characteristics and outcomes. Oncotarget. 2012 3:1566-75. doi: 10.18632/ oncotarget.716. 